\title{
"Hacer escuela, hacer colectivo". Movimientos sociales, jóvenes y experiencias educativas desde una perspectiva antropológica (Rosario, Argentina)
}

\author{
MARILÍN LÓPEZ FITTIPALDI
}

UNIVERSIDAD NACIONAL DE ROSARIO (UNR), ROSARIO/SANTA FE, ARGENTINA

HTTPS://ORCID.ORG/0000-0003-4I82-2652

\section{Introducción}

En las últimas décadas, la problemática educativa se ha colocado en el centro de buena parte de las luchas y procesos de organización colectiva impulsados desde los sectores populares en América Latina (Santillán 2015; Sirvent 2005; Torres Carrillo 2017, entre otros). Se trata de demandas por el derecho a la educación que se concretaron tanto en reclamos en pos de la inclusión y del acceso a la escolaridad, así como en la creación de iniciativas propias en el seno mismo de los movimientos y organizaciones sociales y políticas. Experiencias tales que, al mismo tiempo que visibilizan la persistente continuidad de las desigualdades que atraviesan los procesos de escolarización de vastos conjuntos poblacionales, contribuyen a renovar el debate público en torno a la relación entre educación y política.

En Argentina, entre las iniciativas más resonantes pueden considerarse los denominados "Bachilleratos Populares": escuelas secundarias para jóvenes y adultos/as, orientadas fundamentalmente a sectores populares urbanos que no logran completar sus estudios dentro del sistema educativo oficial. Sus impulsores son diferentes colectivos sociopolíticos - movimientos sociales, cooperativas, partidos políticos - que, en general, inscriben sus prácticas en contextos de pobreza y desigualdad. Según ha sido señalado, pretenden atender a situaciones de "riesgo educativo" y, a la vez, promover procesos de 
“organización y participación comunitaria”, "en clave de educación popular" (Elisalde 2008:93). Desde su primera aparición en el año 2004, se expandieron con una intensidad llamativa, principalmente en Capital Federal y provincia de Buenos Aires, pero también a otros lugares del país ${ }^{1}$.

En un sentido amplio, estas propuestas se enraizaron en experiencias educativas previas gestadas en el campo de los movimientos sociales y organizaciones populares, de larga data en la región (Santillán 2015). Pero, al mismo tiempo, renovaron dicho campo al demandar ser reconocidos dentro del sistema educativo oficial, es decir, la posibilidad de otorgar títulos de acreditación del nivel y, en algunos casos, recibir financiamiento por parte del Estado.

En este contexto se ha ido abonando el interés para un creciente número de investigaciones sobre la temática, desde distintos campos disciplinares y perspectivas teóricas, que han contribuido a conocer los fundamentos y principios orientadores de los proyectos político-educativos (Ampudia 2013; Burgos et al. 2008; Elisalde 2008; Torres Carrillo 2017, entre otros).

Sin embargo, en los últimos años, algunos trabajos han cuestionado el sentido a priori que suele darse a estas caracterizaciones, y han avanzado, de modo concomitante, en reconocer la distancia que existe entre los propósitos que orientan los proyectos educativos y los sentidos que construyen los y las estudiantes (Gluz 2013; Kriger y Said, 2015; Said 2018). Por su parte, los abordajes desde la investigación etnográfica, aunque más escasos, han realizado significativos aportes para complejizar la compresión de estas particulares experiencias educativas. Estos estudios contribuyeron a destacar las tensiones y heterogeneidades al interior de las mismas, así como su carácter dinámico, en la trama de múltiples relaciones con la educación oficial y el Estado (Caisso 2014; García 2011, 2018).

Es posible señalar, no obstante, que las vivencias de las y los estudiantes en el transcurrir por estas escuelas continúa siendo un área relativamente poco explorada, en especial si se compara con la atención otorgada a las experiencias de las y los jóvenes y su protagonismo político en la escuela común (Kriger y Said 2017; Núñez y Litichever 2016; Weiss 2015, entre otros). Lo que es más, aún cuando algunas investigaciones comienzan a problematizar dichas experiencias, lo hacen desde un lugar secundarizado, más para mostrar los límites que enfrentan los proyectos educativos en su realización que, como diría Rockwell (2018b:239), para observar a estos/as jóvenes "como seres humanos en proceso de tomar decisiones y de reapropiarse la experiencia vivida"2.

Este artículo pretende ser un aporte en esa dirección. En concreto, me propongo como objetivo dar cuenta de los proyectos educativos emergentes como una continúa construcción, y en paralelo, resituar a las y los jóvenes estudiantes como sujetos y hacedores clave en el proceso de esa producción colectiva. Propongo que la gestión social, en tanto categoría construida socialmente, puede tomarse como una clave analítica desde la cual entender una trama de prácticas, sentidos y relaciones que configuran el hacer colectivo que da existencia a la escuela. Desde la perspectiva de las y los jóvenes estudiantes, este

\footnotetext{
1 De acuerdo a un relevamiento realizado a nivel nacional, para el año 2015 existían en la Argentina un total de 93 Bachilleratos Populares: el 38\% localizado en la Ciudad de Buenos Aires, el 54\% en la Provincia de Buenos Aires y el 8\% en el resto de las provincias del país. Del universo de 86 experiencias incluidas en el relevamiento, el 46\% están oficializadas y pueden emitir títulos de estudios secundarios. De éstas, 23 se encuentran en la Ciudad de Buenos Aires, 15 en la provincia de Buenos Aires y sólo 2 en el interior del país (Grupo de Estudios Sobre Movimientos Sociales y Educación Popular 2016).
}

2 Como excepciones puede mencionarse a los trabajos de García (2018) y Caisso (2017). 
hacer constituye una experiencia formativa (Rockwell 2012), de la que irán apropiándose en el devenir de la experiencia escolar, en un proceso no exento de tensiones.

\section{Acerca del enfoque teórico-metodológico de la investigación}

El análisis que presento es resultado de una línea de investigación que vengo desarrollando desde el año 2011 hasta la actualidad, orientada a conocer los procesos de configuración de una experiencia educativa impulsada por una organización política, en un contexto barrial de pobreza urbana y desigualdad de la ciudad de Rosario (Santa Fe, Argentina).

Se trata de una escuela secundaria pública, de la modalidad de Educación Permanente para Jóvenes y Adultos (EPJA) 3 . Posee una organización graduada en cinco niveles - denominados "años" -, concentrados a lo largo de tres años calendarios, y funciona en el denominado turno vespertino - de 19 a 21 horas. Si bien se encuentra en funcionamiento desde el año 2011, no fue hasta el año 2018 que obtuvo reconocimiento oficial por parte del Ministerio de Educación Provincial, tras extensos procesos de demanda y negociación impulsados desde la escuela.

La indagación se orienta desde un enfoque antropológico relacional, que privilegia el conocimiento de la cotidianeidad social, pero en articulación con procesos socio-históricos que corresponden a distintas escalas (Achilli 2005). La perspectiva teórica se nutre, principalmente, de los conocimientos elaborados dentro del campo de la etnografía educativa latinoamericana (Achilli 2018; Rockwell 2000), así como de una perspectiva antropológica de la política, que destaca su carácter relacional y en imbricación con otras dimensiones de la vida social (Cañedo Rodriguez 2011; Gledhill 2000; Manzano y Ramos 2015).

Desde estos puntos de partida, y recuperando, a su vez, elaboraciones en el cruce de la antropología y la geografía crítica, propongo pensar la escuela como un "lugar" (Ingold 1993; Massey 2005), en términos de Manzano (2016: 61), "como punto y momento de articulación de una colección de trayectorias y como foco para la producción de nuevos espacios y vinculaciones sociales". Retomando a Santillán $(2015,2019)$, considero a la escuela como un proyecto educativo emergente, y en tal sentido, como una construcción "no acabada", sujeta a significativas variaciones en el curso de sus acciones a través del tiempo.

El proceso de indagación se desarrolló en dos momentos. El primero, coincidente con los primeros tiempos desde la creación de la escuela, entre los años 2011 y 2014. El segundo, desde el año 2016 a la actualidad ${ }^{4}$. Ello influyó en el modo en que se fue elaborando la construcción de la problemática,

3 La Educación Secundaria en Argentina, obligatoria desde el año 2006 (Ley de Educación Nacional No26206), se corresponde solo aproximadamente con el Ensino Medio del sistema escolar brasileño. Tiene una duración de cinco o seis años, según la modalidad orientada o técnica respectivamente, y está destinada a adolescentes y jóvenes que hayan cumplido con el nivel de Educación Primaria. La modalidad de adultos, por su parte, destinada a quienes no han cumplido el nivel en la edad teórica (de 13 a 18 años), recibe a estudiantes de 18 años o más, y se concentra en tres años calendarios.

4 El primero se corresponde con la Tesina de Licenciatura (López Fittipaldi 2015), cuya continuación es el Proyecto de Investigación doctoral: "Movimientos sociales, jóvenes y educación. Un análisis antropológico de los "Bachilleratos Populares" como experiencias socioeducativas emergentes en contextos de desigualdad social” (2016-2021). El proyecto cuenta con el financiamiento de una Beca Doctoral CONICET. 
atendiendo al complejo cruce de temporalidades propias de los procesos sociales estudiados, y aquellas que constituyeron el mismo proceso de investigación (Cerletti, Santillán 2015).

La información empírica se recabó a partir de un trabajo de campo etnográfico, prolongado y en profundidad, que incluyó observaciones de la jornada escolar y entrevistas con docentes y estudiantes en curso y egresados/as. Durante la jornada escolar mantuve frecuentes conversaciones informales con docentes y estudiantes que se encuentran cursando los distintos años, que aun cuando no fueron entrevistas pautadas previamente, permitieron ampliar la información en profundidad, y lo que es relevante, de modo contextualizado. Por último, relevé fuentes documentales de distinto tipo: leyes y normativas nacionales y provinciales, noticias periodísticas y documentos elaborados por la organización.

\section{Procesos de construcción de la escuela: Del freno a los desalojos a la gestión social}

La escuela sobre la que se centra esta investigación nació, como se señaló, en el seno de una organización política. La misma había surgido por la iniciativa de un grupo de jóvenes estudiantes que, en el contexto abierto en los años posteriores a la crisis desatada en el inicio de siglo en Argentina, pretendían impulsar formas de acción política que recuperaran y a la vez renovaran las experiencias de movilización recientes (Documento de la organización, año 2006) ${ }^{5}$. Como puntapié para ese proyecto, en el año 2005, se propusieron impulsar propuestas educativas con niños/as y jóvenes de sectores populares de la ciudad.

El lugar elegido fue un barrio ubicado en el extremo noroeste del municipio, delimitado hacia el este por una ruta provincial y surcado, de norte a sur, por un canal hídrico ${ }^{6}$. Este curso de agua separa dos áreas relativamente diferenciadas al interior del barrio - usualmente consideradas como "zona urbana" y "zona rural". Sin embargo, y aún cuando estas condiciones se expresan de modo desigual, ambas están atravesadas por carencias materiales, déficit en la provisión de servicios públicos, y han sido acuciadas, históricamente, por dos problemas centrales.

En primer lugar, la ocurrencia de inundaciones, a causa del desborde del canal - mitigadas en los últimos años a partir de la realización de obras públicas de infraestructura. En segundo lugar, la permanente amenaza de desalojo de las familias que allí habitan, debido a las complejas condiciones de propiedad y tenencia de la tierra, y fundamentalmente, a la existencia de un proyecto urbanizador que planeaba desarrollar en la zona un barrio privado.

5 La llamada "crisis del 2001" fue una crisis social, económica, política e institucional que azotó a la Argentina en el cambio de siglo, luego de al menos una década de implementación de políticas de corte neoliberal. Tuvo su momento más álgido en diciembre del 2001, cuando se desató una profunda revuelta popular, seguida por la renuncia del entonces presidente Fernando de la Rúa. Suele considerase como un momento histórico en que se dieron las condiciones de posibilidad para la emergencia de experiencias de movilización colectiva, como los "movimientos piqueteros", las "empresas recuperadas" o las "asambleas barriales", inicialmente vistas como novedosas o potencialmente disruptivas. Las mismas fungieron como inspiración - aun desde posiciones críticas- para multiplicidad de experiencias organizativas en los años posteriores (puede consultarse Giarracca y Teubal 2007; Quirós 2011; Svampa y Pereyra 2003, entre muchos otros).

6 En éste y los párrafos siguientes, se recupera, de modo sintético, información obtenida a partir del trabajo de campo realizado desde la creación de la escuela, en el año 2011, y también una exploración previa, durante el 2010, en el marco del movimiento social y los distintos proyectos que el mismo impulsó en el barrio. El trabajo de campo mencionado incluyó entrevistas, observaciones en jornadas de protesta y recorridas por el barrio, así como la revisión de documentos de difusión elaborados por la organización política. 
Con el tiempo, y en un contexto de escalada del conflicto en torno a las inundaciones, primero, y a los desalojos, después, la organización fue consolidando su presencia en el barrio, e incorporó en un movimiento más amplio a numerosas familias que allí residían. Como parte de ese proceso, se impulsaron fuertes demandas frente al Estado municipal y provincial para lograr una solución definitiva a las problemáticas mencionadas, a la vez que se desplegó una estrategia de activa ocupación de los terrenos en disputa, a través de la creación de emprendimientos productivos - huertas, una cooperativa textil y otra de producción láctea -, una radio comunitaria, y espacios para la realización de deportes y esparcimiento.

Este fue el marco en el cual, en el año 2011, la organización decidió crear la escuela, que se emplazó en un terreno ocupado en la denominada "zona rural", el corazón de las tierras disputadas por el proyecto inmobiliario. El objetivo expreso consistía en reforzar la "barrera" frente a la presión de los desalojos, con la convicción de que sería "más difícil tumbar una escuela, que tumbar casas y nada más" (Laura, docente. Registro de Campo Número 13, entrevista. 04/04/11) ${ }^{7}$.

Sin duda, para entender la gravitación que tomó posteriormente el proyecto educativo, resulta menester considerar también las características de la oferta educativa en la zona. Las escuelas de nivel secundario más cercanas, ciertamente escasas -solo una secundaria orientada y una secundaria de la modalidad de jóvenes y adultos - se encuentran a una distancia que ronda entre los dos y cuatro kilómetros. Pero además de la distancia, un factor importante es que el recorrido implica cruzar zonas reconocidas como peligrosas por los residentes del lugar:

Hay otra escuela que está en otro barrio, que se llama Cristalería. Y bueno, también, por ahí la gente de esta zona se tiene que trasladar hasta allá, y quizás capaz que les sea... tiene que cruzar una ruta, una vía, es mucha travesía... y por ahí la inseguridad puede ser una traba que impide que otros terminen, digamos, que la gente de esta zona pueda terminar la escuela (Ariel, 21 años, egresado. RNo 90, entrevista. 01/12/18).

Sin embargo, es importante remarcarlo, en el momento de su creación, la escuela sería planteada, antes que nada, como una "bandera de lucha" frente a los desalojos:

La escuela nace como una manera de evitar desalojos, que se daban por el tema de las grandes inmobiliarias que querían cooptar todos esos terrenos. Y bueno, era una forma de hacer que estos estudiantes empezaran a organizarse, y empezaran a, que creo que ese es el gran objetivo de la escuela, la organización de los estudiantes, o de la gente que se acerca, para defender lo que sea necesario (Andrea, docente. RNo 23, entrevista. 27/08/18).

\footnotetext{
7 Todos los nombres propios incluidos en este trabajo son ficticios para resguardar el anonimato de los protagonistas. Las frases y palabras encomilladas refieren expresiones literales. Las categorías sociales aparecen en itálicas. De aquí en más utilizo la abreviatura "RN" (Registro de Campo $\mathrm{N}^{\circ}$ ) para indicar la numeración de los Registros.
} 
Fue así que, en ese primer momento, la escuela reunió a personas que se hallaban vinculadas al movimiento social de distintas formas. El cuerpo docente, unas diez o doce personas, según fue oscilando en el tiempo, se conformó a partir de militantes y personas allegadas ${ }^{8}$.

Las y los estudiantes, por otra parte, fueron convocados entre aquellos residentes del barrio que ya formaban parte de otros proyectos impulsados por la organización, o bien que estaban entre aquellas familias más afectadas por el posible desalojo. Esa cohorte inicial contó con unos veinticinco estudiantes, jóvenes y adultos, aunque no todos lograron finalizar. Es así que, en su experiencia, asistir a la escuela se presentaba en un marco de continuidad con la participación en otros espacios impulsados por el movimiento social. Nora, una estudiante egresada e integrante de la primera cohorte, recordaba en una entrevista esos primeros tiempos de la escuela:

Vos tenías que empezar esa escuela sabiendo que capaz que tu título [de egresada] nunca iba a estar. Entonces era bancar la escuela para que no te lleven por delante, o sea, para ganarle a los countries [barrios privados], con una escuela. Como sea. Con título o sin título, lo que más primaba ahí era no permitir el avance de los countries (Nora, 52 años, egresada. RNo 56 , entrevista. 22/12/17).

No obstante, ese momento fundacional fue el puntapié para un largo proceso, no exento de tensiones y desafíos para las y los propios protagonistas. La escuela atravesaría múltiples transformaciones que fueron configurando el proyecto educativo tal como existe actualmente. Así, para cuando reinicié el proceso de investigación, en el año 2016, y como varias veces me fue señalado por las y los sujetos que habían estado desde los primeros tiempos, me esperaba "otra escuela", muy diferente a la que yo había conocido.

Para reseñar brevemente estos procesos, es posible señalar, en primer lugar, que en el año 2013 la profundización de la conflictividad en el barrio obligó a la organización a mudar la escuela a otro sector, ante la ocurrencia repetida de hechos violentos que ponían en riesgo la continuidad del proyecto. La escuela se instaló entonces en la denominada la "zona urbana", más densamente poblada. Esto permitió el acceso a mayor cantidad de personas, quienes encontraban dificultades para recorrer la distancia hasta la antigua ubicación de la escuela.

Como surge de las entrevistas realizadas, las y los estudiantes refieren la peligrosidad de transitar hacia esa zona del barrio, en especial por las noches, que es el horario en que se cursa la escuela. También señalan las dificultades que derivan del estado de gran deterioro de las calles, algunas de tierra, y la ausencia de alumbrado público: "Son veinte cuadras de una zona rural. O sea, calles de barro cuando llueve, de tierra. Donde te empantanas apenas caen tres gotas" (Nora, 52 años, egresada. RNo 56, entrevista. 22/12/17).

8 Hasta el día de hoy la tarea docente es llevada a cabo por personas con distinta formación: profesionales, personas idóneas, estudiantes universitarios/as, y docentes de nivel secundario en actividad o retirados/as. Poseer un título docente no es un requisito excluyente, sino más bien, el compartir cierta "afinidad política" con respecto al proyecto. Se organizan - al igual que en otros Bachilleratos Populares- en "parejas pedagógicas" que suponen la planificación y el dictado de las clases en duplas, o en ocasiones en tríos. A su vez, las y los docentes conforman pequeños equipos que asumen distintas tareas vinculadas a la administración escolar y, junto con la asamblea que se realiza de forma regular, a la toma de definiciones. 
Según relataba otra estudiante egresada, aunque deseaba comenzar la escuela apenas supo de su existencia, esto no le resultó posible hasta que la misma se instaló en la zona urbana, más cercana a su domicilio. Una condición que, en su descripción, compartía con otras compañeras:

Acá es una zona peligrosa, viste, era como que no estaba... si bien lo que nos ofrecía [la escuela] era para nosotras, llegarnos hasta ahí [la zona rural] no era fácil, para nosotras que no tenemos quien nos acompañe y todo eso. Y después bueno, se vino y se mudó acá, consiguió en este lugar, que pudieron alquilar y... y bueno, me acerqué (Laura, 45 años, egresada. RNo 50, entrevista. 31/10/16).

Otro aspecto de importancia para considerar las transformaciones que atravesó la escuela remite al proceso de negociación entablado con el Ministerio de Educación provincial para obtener el reconocimiento oficial. En el curso del mismo, se debieron adoptar, de modo progresivo, diferentes disposiciones ministeriales, referentes al diseño curricular, condiciones edilicias, formas de evaluación y promoción de los estudiantes, entre otras. Aun cuando la lucha por el reconocimiento se extendió más allá en el tiempo, desde el año 2014 el Ministerio concedió a la escuela la posibilidad de entregar títulos oficiales de finalización del nivel, por intermedio de otra institución educativa para adultos.

Estas transformaciones contribuyeron a disipar rumores entre los pobladores que ponían en duda, en los términos expresados por los y las estudiantes, que se tratara de una escuela de "real" o "de verdad":

Cuando empieza en el 2011, [cuando el movimiento] aparece con la escuela, la gente decía que era una escuela como que... entre comillas, jugaban a la escuelita, o sea... qué le van a dar a... qué le van a dar al movimiento, si es una escuela que surgió de un día para el otro, quién la creó, quién la hace, quién la fundó... qué título le van a dar, si quieren estudiar algo..." (Alexis, 21 años, egresado. RNo 90, entrevista. 01/12/18).

Como relataba una estudiante graduada, con el transcurso del tiempo "[los vecinos fueron viendo] que no es una mentira todo lo que está pasando en la escuela. Y que el título es real ¡cada vez que tenemos el título lo mostramos! Esto es real” (Laura, 45 años, egresada. RNo 50, entrevista. 31/10/16).

Por último, cabe mencionar que la propuesta educativa se amplió en el año 2016 incorporando el nivel inicial, con la creación de un jardín de infantes que funciona en la misma locación que la escuela secundaria. Al mismo asisten niñas y niños que habitan en el barrio, frecuentemente familiares de las y los estudiantes del nivel secundario. A diferencia de éste último, el jardín fue oficializado rápidamente, y su existencia aportó a profundizar nexos con instituciones locales, como por ejemplo, el centro de salud barrial, dependiente del estado municipal.

De acuerdo a lo señalado por Nicolás ( $\mathrm{RN}^{\circ}$ 61, entrevista. 04/05/18), docente de la escuela, esta "articulación" habilita a "conocer las particularidades, detectar algo ahí, acercarse al centro de salud, hablar con la referencia en el centro de salud, encontrar un modo de laburo para ese caso puntual, para distintos problemas que puede tener un niño del barrio". Del mismo modo, según pude observar en 
repetidas ocasiones, desde el centro de salud se derivan niños/as para quienes no existen vacantes en otras escuelas de la zona.

En conjunto, estos procesos contribuyeron a que la propuesta educativa del movimiento social fuera cobrando gravitación en el espacio barrial, ampliando su alcance y dimensiones. Esto se acompañó de un importante aumento de la matrícula, especialmente nutrida por jóvenes, que se elevó a cerca de ciento treinta estudiantes. Actualmente, "el promedio de edad es de 20, 22 años. Tenés un par de personas adultas, que te estiran el promedio. Pero que en realidad tienen 20, 22 años la mayoría de los estudiantes" (Nicolás, docente. RNo 61, entrevista. 04/05/18).

No obstante, lo que resultaba más importante desde la perspectiva de las y los docentes, es que en el curso de estos procesos "cambió el estudiante": "Ahora es distinto, son los chicos, son los pibes que uno ve en los barrios. Es así. No son [como] Nora, gente más politizada. [Es] otra construcción. Hay que pensar otras estrategias" (Nicolás, docente. RNo 61, entrevista. 04/05/18).

En contraste con aquellas primeras cohortes, cuya motivación era primeramente "hacer el aguante político", los nuevos estudiantes no tenían contacto previo con la organización, sino que se acercaban, de acuerdo a sus expresos motivos, con el objetivo de terminar sus estudios y obtener el título del nivel secundario. Como insistía el docente entrevistado, "esto de lo asambleario y demás, la gestión social de la escuela, es como que al principio... cuesta” (Nicolás, docente. RNo 61, entrevista. 04/05/18).

\section{“Hacer entre todos”. La gestión social como categoria local}

Desde aquellos locos, chicos y chicas, que con el movimiento, se imaginaron una escuela, una forma de hacerse presentes acá en el territorio, a estos chicos que hoy terminan 5to año, hay, hubo un elemento que nos une a todos, que es el hacer colectivo, eso que nosotros definimos en dos palabras: gestión social (Fernanda, docente. RNo 88, Acto de cierre del ciclo lectivo y entrega de libretas. 08/12/2018).

Luego de un tiempo sin asistir a la escuela, me disponía a comenzar una nueva temporada de trabajo de campo. Andrea, una de las pocas docentes que aun permanecían desde la creación de la misma y a quien, por ese motivo, yo conocía bien, fue la encargada de recibirme en su clase de primer año. Después de presentarme a las y los estudiantes Andrea propuso que me cuenten, "un poco como repaso para todos nosotros", aclaró, cómo era la "dinámica de trabajo" en la clase de integración.

No le resultó fácil, al comienzo, que algunos/as estudiantes dejaran de escribir en sus carpetas, preocupados/as como estaban por terminar un trabajo de la clase anterior. Tras insistir, las y los estudiantes comenzaron a participar más activamente. "Cuéntenle un poco a Marilín, ¿de qué se trataría la integración?”, preguntó Andrea. Algunas estudiantes fueron arrojando respuestas: “de comunicarnos", "de hacer trabajos entre todos", "participar en clase". La docente insistió:

Andrea: ¿Pero de qué hablamos más que nada en integración? [Hace una breve pausa, esperando respuesta] Che, ¿les comieron la lengua los ratones?

Marcos: La gestión social. 
Andrea: ¡De la gestión social, muy bien Marcos! ¿De qué se trataría la gestión social?

Marcos: Es una gestión social... [Sus compañeros se ríen, y entonces el estudiante reformula] que lo hacemos en grupo...

Graciela: Que opinamos todos.

Martín: Que ninguno queda afuera.

Andrea: ¿Y que hicimos el último viernes, que fue muy importante?

Noelia: ¡Una asamblea!

Andrea: Una asamblea [con tono de aprobación]. ¿Y de qué se trata la asamblea, para los que no estuvieron? ¿Quién puede contar un poco de la asamblea?

Noelia: Hablar de las distintas opiniones que tenemos acerca de las materias, de los profesores, de los compañeros... de la escuela... de todo.

Gustavo: Qué les gustaba y qué no.

Andrea: Aja. Porque además, también hicimos como un esquema de organización, ¿̇se acuerdan? ¿En qué tratábamos de organizarnos?

Gustavo: El horario de entrada a la escuela y la salida.

Mara: Armar y desarmar el salón.

Este punto generó algunas controversias, y las y los estudiantes empezaron a lanzar acusaciones cruzadas acerca de quiénes se habían quedado más o menos veces, y quienes, a los ojos de sus compañeros/as, "no hacen nada". La docente intervino tratando de poner orden, señalando la importancia de organizarse y "que se vayan rotando", "para que no pase esto". A continuación destacó un último punto de acuerdo, el mate': insistió en los momentos de la clase adecuados para prepararlo, y la importancia de que todos/as aporten algo de dinero para comprar la yerba y el azúcar. "Ahora vamos a empezar a trabajar”, indicó por último, dando cierre a este intercambio e iniciando, según interpreté en ese momento, el tiempo de la clase (RNo 63. Clase de 1er año. 29/05/18).

No era la primera vez que escuchaba el término gestión social, expresión que, con el correr del tiempo, había ido adquiriendo centralidad en la descripción que las y los sujetos hacían de la escuela. Era, en primer lugar, una categoría elaborada históricamente en relación al Estado y las políticas educativas. Esta figura, incorporada por primera vez en la Ley de Educación Nacional (No26206) del año 2006, reconoció legalmente -junto a las dos modalidades vigentes de gestión, privada y estatal- a las iniciativas educativas impulsadas por organizaciones de la sociedad civil (Art. 13 y 14$)^{10}$. Sin embargo, dado que son finalmente las provincias las que deben "autorizar, reconocer, supervisar y realizar los aportes correspondientes" a las instituciones educativas (Art. 140), y en tanto la provincia de Santa Fe no completó a la actualidad la elaboración de una nueva Ley de Educación provincial, en adecuación a la Ley nacional, la figura es inexistente a nivel de la jurisdicción.

9 Es una infusión cuyo consumo está muy extendido en la Argentina y otros países de la región.

10 De acuerdo a los lineamientos elaborados a nivel nacional, que deben ser, no obstante, refrendados a nivel provincial, se define a las escuelas de gestión social como aquellas que "prestan servicios educativos universales y gratuitos y son gestionadas por organizaciones sociales"; se orientan a "población en situación de vulnerabilidad social" desarrollando acciones para la "inclusión y permanencia de esos grupos en el sistema educativo"; y promueven "una gestión comunitaria en lo que hace al gobierno escolar" (Resolución N³3/07. Anexo 01). 
Apelar al término gestión social para describir la escuela era, de este modo, legitimar el proyecto educativo en base a la letra de la ley nacional, y a la vez abrir el juego para dotar a la categoría con sentidos propios. De hecho, la organización había participado activamente de los debates orientados a formular la nueva ley en el contexto provincial, presionando para la inclusión de la categoría y apuntando elementos para su definición.

Así, desde la perspectiva de las y los impulsores del proyecto, como Pedro, referente político de la organización, la gestión social debía fundarse en la "innovación pedagógica" y "la gestión escolar en forma horizontal, democrática, asamblearia" ( $\mathrm{RN}^{\circ}$ 49, Debate por una Ley Provincial de Educación). En palabras de Nicolás ( $\mathrm{RN}^{\circ}$ 61, entrevista. 04/05/18), ser “asamblearia, gratuita e inclusiva”, una "prefiguración" de la educación por venir: "Nosotros creemos que la gestión social en educación, es la educación del siglo XXI. Donde los estudiantes toman decisiones de lo que pasa dentro de la escuela. Entre otras cosas, digo, ese es como el pilar fundamental".

Ahora bien, volviendo a la situación de campo con la que inicié este apartado, lo que captó mi atención fue el modo en que la categoría era puesta en uso por las y los sujetos, designando un conjunto de prácticas diversas, desplegadas en la cotidianeidad de la experiencia escolar: modos de trabajo e interacción en el aula - "hacer en grupo", "participar", "opinar"-, formas de organizarse y mecanismos para la toma de decisiones - "la asamblea" - y actividades necesarias para el funcionamiento diario de la escuela - "el armado del salón", "horarios de entrada y salida de la escuela”. Prácticas éstas que las y los sujetos a cargo del proyecto suelen sintetizar como un "hacer entre todos esta escuela que queremos" (Andrea, docente. RNo 72, entrevista. 10/09/2018).

Asumiendo el término gestión social, entonces, no como una definición cristalizada, sino como una categoría social e históricamente construida en el proceso de hacer la escuela, me propuse intentar asir aquello que era pasible de ser concebido o, más precisamente, experimentado, como parte de esa gestión social. Esto me orientó a observar más detenidamente multiplicidad de acciones - prácticas, expresiones verbales, interrelaciones - que día a día se desarrollaban en la escuela, y que tenían por protagonistas tanto a las y los docentes como a las y los estudiantes. Considerando el "contexto de situación" - expresión evocada por Peirano (2002) en referencia a Malinowski -, en el que la categoría emergía, es decir, a modo de "repaso" de lo trabajado en las clases, me pregunté también por la gestión social como algo que debía ser aprendido por las y los estudiantes.

De este modo, en los apartados que siguen, propongo explorar el término gestión social como una clave para acceder al modo en que se despliega el hacer colectivo que da existencia a la escuela. Esto es, como plantea Quirós (2014: 52), la posibilidad de acceder a una perspectiva local no tanto como punto de vista intelectual, sino como "forma/s y posibilidad/es de hacer, producir y crear vida social". Un hacer que será tensionado, resistido y apropiado como parte del proceso escolar.

\section{La gestión social en la experiencia escolar cotidiana}

Para las y los estudiantes, el camino de ir desentrañando de qué se trata la gestión social comienza desde el momento que ingresan a la escuela. Cada año, el primer día de clases se realiza una asam- 
blea en la que participa la totalidad del alumnado y del cuerpo docente. La asamblea era un mecanismo utilizado en la escuela desde su creación, la cual se suponía un espacio de deliberación y toma de decisiones conjuntas.

Sin embargo, en la actualidad, como reconocían las y los docentes, "no es tan fácil que los estudiantes entiendan qué es una asamblea. Los que están en 4to la tienen más claro. [...] Se va mamando con el correr de los días" (Nicolás, docente. RNo 61, entrevista. 04/05/18). Por ese motivo, con las y los ingresantes de 1ero, el primer mes del año se dedica a entrevistas individuales, destinadas a "conocer a cada uno de los estudiantes, y que los estudiantes conocieran un poco, de que se trataba la escuela" ( $\mathrm{RN}$ o 63. Observación en clase de 1er año. 29/05/18).

Además, como se menciona en el apartado anterior, también se trabaja sobre la gestión social en la clase de integración que, a diferencia de la asamblea, había sido creada en tiempos recientes. Con un dictado semanal, tiene el propósito de articular los contenidos de las distintas asignaturas, y particularmente en el primer año, trabajar en la "construcción y fortalecimiento del grupo" (Andrea, docente. $\mathrm{RN}^{\circ}$ 72, entrevista. 10/09/2018). En cambio, en 5 to año la clase de integración se destina, casi exclusivamente, a la organización del festejo de graduación, y a dirimir los conflictos que, como veremos más adelante, este proceso acarrea.

Por otra parte, más allá de estos momentos de trabajo específicos, la gestión social es un desafío que se pone en juego en el día a día de la escuela. Según fui registrando al asistir semanalmente, cada jornada escolar debía comenzar y terminar con el armado y desarmado del salón. En tanto por las mañanas funciona en el mismo espacio el jardín de infantes, se requería disponer diariamente las mesas y sillas a utilizar, para luego retirarlas y llevarlas al lugar reservado para su guardado. También era necesario realizar la limpieza diaria del lugar, junto a trabajos de mantenimiento de lo más diversos: cambios de luminarias, reparaciones en el mobiliario y las instalaciones e, incluso, tareas de jardinería en el patio escolar. La distribución de estas tareas debía realizarse cada día, "sin que haya un orden establecido, porque se intentaron hacer listas que ordenen... los lunes, los martes... y no, nunca se logra" (Andrea, docente de 1er año. RNo 72, entrevista. 10/09/2018).

En las clases de 1er año la distribución y realización de tareas no sucedía sin tensiones. Con respecto al armado del salón, eran los/as primeros/as estudiantes en llegar a clases quienes debían encargarse. Con el correr de las semanas se fue reduciendo la cantidad de estudiantes que llegaban a horario, lo que postergaba más y más el inicio de la clase. A veces, el pequeño grupo de estudiantes que sí llegaban a tiempo permanecían afuera, rehusándose a entrar a la escuela, a la espera de más compañeros/as.

Como consecuencia, terminaban por tomar la tarea las docentes, junto a dos o tres estudiantes, o con la colaboración de estudiantes de los cursos superiores, quienes ya habían cumplido con el armado del propio salón de clases. Esta situación, ante el evidente contraste entre cursos, iba generando creciente malestar con el transcurrir del año: ‘ $¡ A$ ver si podemos mejorar los horarios en 1 ero! $i L o s$ de 3ero y 5 to ya están hace rato!' (Tamara, docente. RNo 75. Clase de 1er año. 17/09/2018); “iEs siempre lo mismo! ¡Yo ya no sé qué hacer con el tema del salón!" (Tamara, docente. RNo 76, clase de 1er año. 24/09/2018).

Al finalizar la jornada, nuevamente debían asignarse responsables de ordenar y limpiar los salones. Ante la insistencia de las docentes con advertencias tales como "ahora no nos vayamos todos cor- 
riendo”; “ $i$ no nos olvidemos de que hay que limpiar el pizarrón!” (Andrea, docente. RN 69. Clase de 1er año. 27/08/18), emergían las acusaciones entre compañeros/as: “iAlgunos compañeros nunca se quedan!” (Estudiante. RNo 65. Clase de 1er año.11/06/2018); “iYo me quedo siempre!” (Estudiante. RNo 73. Clase de 1er año.10/09/2018). Sin embargo, a diferencia del momento de inicio de la jornada, al hallarse todos/as presentes se lograba mayor circulación en la asignación de responsables. De este modo, si el armado del salón recaía, por lo general, en las mismas tres o cuatro personas, hasta los más renuentes debían implicarse de cuando en cuando en lo que respecta al desarmado.

Hacia el mes de octubre, acercándose al final de la cursada, las docentes optaron por suspender el horario de recreo, para recuperar el tiempo perdido al inicio de la clase, pero también como llamado de atención a las y los estudiantes. La reacción por parte de éstos no se demoró en llegar: al grito de "recreo" se prepararon para salir del aula, siendo interceptados por Tamara, la docente:

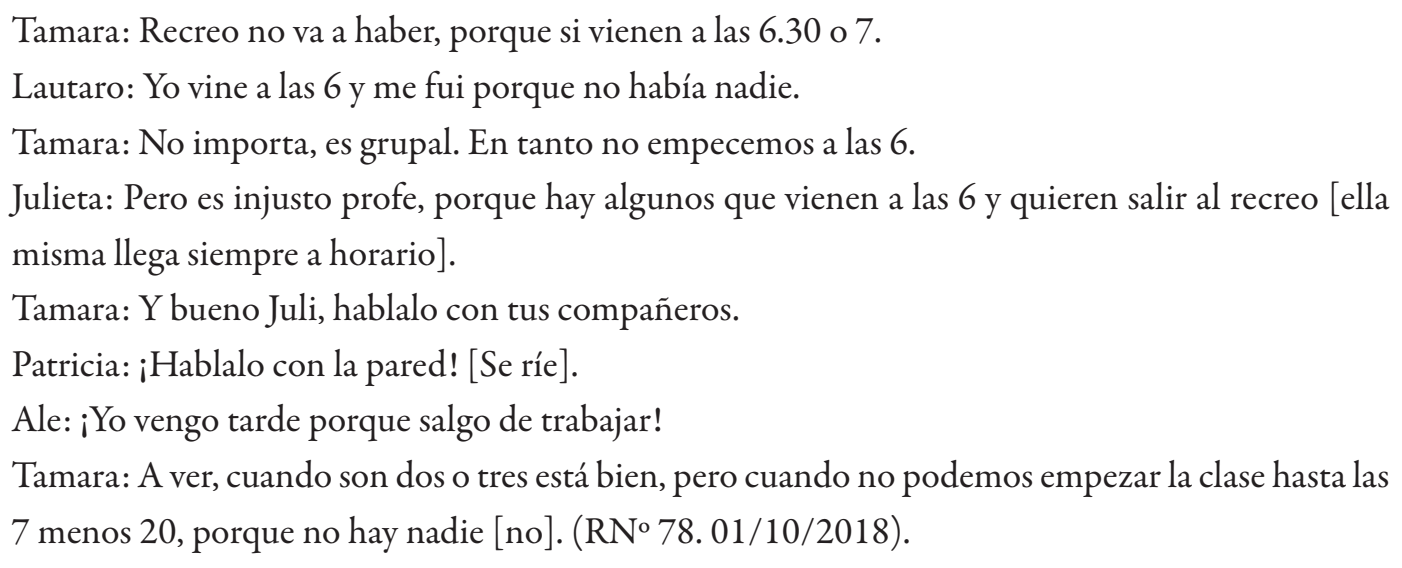

La docente interrumpió allí la discusión y dio inicio al tema que trabajarían en la clase. La suspensión del recreo se mantuvo durante semanas, pero no logró modificar el comportamiento de los y las estudiantes. De este modo, hasta fin de año no se logró mejorar sustancialmente la puntualidad, tampoco la distribución de tareas en lo que atañe al armado y desarmado del aula.

A razón de la gestión social de la escuela, también se requería el compromiso de las y los estudiantes en tareas que suelen estar, en otras escuelas, a cargo del personal de la misma. Tal es el caso del control de asistencias. Así, durante una clase, las docentes preguntaban por los estudiantes que se habían estado ausentando mucho, generando la reacción de una de las estudiantes, quien reclamó que las asistencias no eran debidamente controladas:

Antonela: ¡Si no nos ponen presente!

Tamara (docente): Bueno, está bien. ¡Pidan, pidan su presente! ¡La escuela también se autogestiona! Antonela: [con fastidio] ¡Si nos acordamos, nos acordamos, pero tienen que anotar!

Tamara (docente): Chicos, piensen esto. En las otras escuelas hay preceptor, hay portero, está el docente... esta escuela es una escuela de gestión social, entonces entre todos coordinamos algunas cosas. Esto es para nosotros tener un registro, pero también para ustedes. Entonces, che, hay que tomar asistencia, puede tomar de hecho alguno de ustedes asistencia. 
Andrea (docente): Claro, y no es de botón, vos decís, vamos a botonear... es para ver que le está pasando a tal compañero que hace una semana o diez días que no viene ${ }^{11}$.

La estudiante ya no intervino más y permaneció en silencio, aunque evidentemente por su expresión, poco conforme con la respuesta que recibió por parte de las docentes ( $\mathrm{RN}^{\circ} 51$, clase de 1er año. 31/10/2016). De hecho, durante las clases observadas la asistencia nunca fue tomada por los estudiantes, siendo una responsabilidad asumida por parte de las docentes. Sin embargo, el momento de control de asistencias resultaba una instancia donde las docentes involucraban a los y las estudiantes presentes, preguntando acerca de los motivos por los cuales sus compañeros se hallaban ausentes, y comprometiendo, en ocasiones, a los estudiantes a acercarse a los domicilios de sus compañeros para conversar con ellos.

Por último, otra de las tareas asociadas a la gestión social que deben asumir las y los estudiantes es la realización de actividades para la recaudación de dinero, destinado a la compra de bienes de consumo diario, o bien a eventos o salidas escolares. Aunque para afrontar los gastos más importantes -como el pago del alquiler, impuestos y servicios- existen otras estrategias encaminadas por docentes y referentes del proyecto escolar, los fondos requeridos para gastos menores se recaudan a través de actividades como la venta de rifas, o la organización de bingos, de las que se espera que participen todos los integrantes de la escuela ${ }^{12}$. Como pude observar durante el trabajo de campo, no era poco el tiempo destinado a la organización de dichas actividades, que podía extenderse durante semanas, para lo cual se utilizaba tiempo al comienzo de cada clase. Si bien eran los docentes quienes tomaban a su cargo la coordinación de las mismas, se incentivaba permanentemente la participación de las y los estudiantes.

Así, arribando al último trimestre del año, se había organizado una rifa con el objetivo de reunir fondos para los festejos de fin de curso. En una de las clases observadas, una de las docentes comentó a los y las estudiantes, entusiasmada, la importante recaudación lograda. Éstos/as últimos/as habían participado vendiendo los números del sorteo, y reuniendo los elementos para el premio, una canasta de alimentos. "Está bueno que cuando hay una cosa así colaboremos todos. ¡Felicitamos a todos aquellos que se esforzaron!”.

Como el sorteo se había realizado durante un bingo, en esta ocasión organizado por el jardín de infantes, la docente agregó a continuación: “También quiero felicitar a Caro y a Patricia que vinieron al bingo" (Andrea, RNo 75, Observación en clase de 1er año. 17/09/18). Si de la venta de rifas participaban casi todos/as los estudiantes, no sucedía lo mismo con la asistencia a eventos que, como el bingo, se realizaban por fuera del horario escolar y eran organizados por otros cursos, o bien, como en este caso, por el jardín de infantes.

11 "Botón" es, en lenguaje popular, quien comete la acción de delatar (o "botonear").

12 Hasta el momento, y aun habiendo avanzado en el reconocimiento oficial, la escuela no recibe los aportes económicos con los que se había comprometido el gobierno de la provincia. Además de recibir donaciones individuales, las fuentes de ingreso de dinero incluyen subsidios municipales o provinciales a través de la presentación de proyectos específicos, o bien proyectos de vinculación con la Universidad (RNo 95. Asamblea de docentes. 29/08/2019). Sin duda, la planificación, presentación e implementación de tales proyectos puede considerarse parte de la gestión social de la escuela. No obstante, aquí no me detengo en ello ya que las y los estudiantes no participan de estas tareas. 
En esta oportunidad, por ejemplo, de ese curso habían asistido solo dos estudiantes. No obstante, la docente aprovechó la ocasión para familiarizarlos con el funcionamiento del jardín, un espacio en el que siempre se intentaba fomentar la participación de los estudiantes en curso y egresados: “Y siempre se necesita gente que quiera venir a colaborar [...] ivan a ver qué bien que la pasan!”. Luego de este intercambio, dos estudiantes se comprometieron a asistir (Andrea, docente. RN 75. Clase de 1er año. 17/09/2018).

En suma, mientras un conjunto variable de estudiantes se va involucrando activamente, algunos/as haciendo el esfuerzo de montar y desmontar el aula, otros/as recaudando el dinero necesario para el sostenimiento de la escuela, o bien, asistiendo al jardín de infantes para colaborar, un número no despreciable de estudiantes se resisten a realizar las tareas que deben cumplir, desencadenando fricciones entre sí y con las y los docentes.

Así, a partir de las situaciones descriptas, es posible ver la cotidiana puesta en práctica de la gestión social como un proceso en permanente construcción, que no se sucede sin tensiones, y en el que tanto docentes como estudiantes asumen un lugar activo. De un lado, las y los docentes pretenden imprimir direccionalidad a los aprendizajes, otorgando un tiempo significativo en cada jornada a recordar e insistir sobre la gestión social, reforzando sentidos y orientando las prácticas. Del otro, las y los estudiantes expresan, de maneras más o menos explícitas, las tensiones que experimentan frente a una propuesta escolar que perciben diferente y que no acaban de comprender. La resistencia, siguiendo a Rockwell (2018a), puede entenderse como una fuerza que se opone a la fuerza activa expresada en las acciones escolares, y que emerge en la tarea de encontrar sentido a su experiencia cotidiana.

\section{La gestión social entre tensiones y apropiaciones}

Ahora bien, ¿qué sentidos construyen las y los estudiantes en el curso de estas experiencias cotidianas en la escuela? ¿Qué apropiaciones se producen a través del tiempo? En este apartado me interesa profundizar en los sentidos construidos por las y los estudiantes en torno al aprendizaje de la gestión social como parte del proceso escolar. Para ello, me centro en el relato que realizan de sus experiencias en la escuela jóvenes egresados/as, o que se encuentran finalizando el último año y están próximos a graduarse.

En las entrevistas realizadas surgía, de modo frecuente, un elemento en común: la inicial dificultad para comprender aquello que resultaba "extraño", comparado con otras escuelas por las que los y las estudiantes habían transitado. En sus palabras, “iTodo con nombres que nada que ver! Que no estaban en la [escuela]... y era como que... bueno... empezar a entender... y encima ellos lo que te decían es que no te daban estudios así... como la escuela” (Analía, 24 años, egresada. RNo 91, entrevista. 29/08/2019); "Primero se me hizo medio extraño, porque nada que ver con las otras escuelas. Pero a medida que iba viniendo me iba copando, así que me re gustó, y me quedé” (Ileana, 29 años, estudiante de 5to año. RNo 85, entrevista. 12/11/18).

Como reflexionaba Ariel, un estudiante egresado, recordando sus primeros pasos por la escuela: 
ler año fue un proceso de adaptación para mí, fue una experiencia bastante... quien dice, de alguna manera, emocionalmente, tuve crisis, porque me tuve que adaptar a todo este modelo de esta escuela, y había siempre... como que me alteraba, me ponía nervioso a la vez que los profesores me querían hacer participar. Entonces yo me enojaba (Ariel, 21 años, egresado. RNo 90, entrevista, $10 / 12 / 18)$.

Sin embargo, el mismo joven destaca como esa experiencia se fue transformando con el correr del tiempo, de modo que ya "en el segundo año de cursado, yo ya estaba más adaptado, iba comprendiendo más":

Desde 1er año a 5to año, cuando ya llegue a 4to, 5to, que vendría ser cursando el ultimo año acá en la escuela, creo que ya estaba bastante labrado, trabajado, digamos, por la escuela, por todos los profesores, y ya me sentía más en familia, y venía con más ganas. Digamos, no solo porque ya estaba terminando, sino porque esa adaptación, esa experiencia nueva yo ya la tenía procesada e internalizada (Ariel, 21 años, egresado. RNo 90, entrevista, 10/12/18).

Ya sea como "adaptación" o "internalización", en los términos que lo expresa Ariel, o como "transformación”, en las palabras de Analía - “a mí me transformó una banda” (Analía, 24 años, egresada. RNo 96, entrevista. 29/08/2019) - o bien como "cambios en la personalidad", como se enuncia en el pasaje citado más abajo, las reflexiones de estos/as jóvenes remiten a experiencias profundamente formativas, es decir, en las que sedimentan apropiaciones cognitivas y vivenciales duraderas (Rockwell 2012:711).

Se trata, a la vez, de procesos que se van desenvolviendo lentamente, con el transcurrir de la experiencia escolar. $\mathrm{Al}$ rememorar su paso por la escuela, Carolina señala que al comienzo "no había escuchado ni del movimiento, menos de la escuela. “'[...] yo acá entré y les dije: ¡a mí no me joden con la política! ¡Y después yo sola me fui involucrando en todo!”. Más adelante agrega: “y bueno, se fueron pasando los años, que sé yo. Cada vez me enamoraba más la escuela. ¡Era algo muy zarpado! [...]. Ya de tercero, a cuarto y quinto, me fue cambiando toda la personalidad, digamos" (Carolina, 20 años. $\mathrm{RN}^{\circ}$ 71, entrevista. 03/09/2018).

A continuación, propongo observar la puesta en práctica de estos aprendizajes a través de un proceso que aparece siempre en las narraciones de las y los estudiantes y egresados/as como muy significativo, cargado, a la vez, de tensiones y expectativas. Me refiero a la organización del festejo de graduación.

En las observaciones en clases de 5 to año no registré, a diferencia de lo descripto con respecto al 1er año, conflictos en torno al armado y desarmado del salón, ni tampoco ocasiones en que los y las docentes debieran recordar los acuerdos en torno a la gestión social. Incluso, como se trasluce en algunos de los fragmentos de registros de campo citados en el apartado anterior, en ocasiones la comparación con el comportamiento de las y los estudiantes de los cursos superiores se ponía de relieve cuando las docentes regañaban a los estudiantes más novatos. En cambio, era la graduación el tema sobre el que giraban todos los conflictos. 
Buena parte de las tensiones emergían debido a que, para poder llevar la graduación a cabo, las y los estudiantes debían recurrir a distintas estrategias aprendidas como parte de la gestión social. Por ejemplo, la organización entre todos/as de actividades para la recaudación de dinero para solventar los gastos, como ser la realización de bingos, puestos de venta de comidas en los horarios de recreo e ingreso a la escuela, o la realización de rifas.

La graduación fue un momento de, por ejemplo, pensar que se hacía para recaudar plata. Primero tuvimos que pensar que íbamos a hacer para recaudar plata, que lugar íbamos a alquilar [...] Por ahí había desacuerdos, por ahí no nos podíamos organizar bien para hacer algo. A veces había, quizás, falta de comunicación, digamos. La falta de comunicación de algunos compañeros (Ariel, 21 años, egresado. RNo 90, entrevista, 10/12/18).

Como expresa Ariel, la puesta en marcha de estas iniciativas frecuentemente acarreaba discusiones entre las y los estudiantes acerca de las responsabilidades asumidas. Para dirimir estos conflictos, tal como ocurría cuando se trataba de actividades involucradas en la gestión social de la escuela, las y los estudiantes contaban con las clases de integración y a las asambleas, coordinadas por las y los docentes.

Eventualmente, las tensiones se resolvían, y las actividades terminaban realizándose: "Llegó un momento que pudimos ponernos de acuerdo, que pudimos terminar de organizarnos" (Ariel, 21 años, egresado. RNo 90, entrevista, 10/12/18). Cabe destacar, a su vez, que a pesar de los reproches que circulaban entre compañeros/as, la organización de la graduación iba acompañada de un importante involucramiento, y como a veces señalaban las docentes, hasta aquellos estudiantes que más se ausentaban a clases, luego "iban a la escuela para hacer la graduación" (Andrea, docente. RNo 74, entrevista. 17/09/18).

Del mismo modo, en las observaciones y entrevistas realizadas con los estudiantes de 5to año registré como las tensiones se iban acumulando a medida que se acercaba la fecha del esperado evento desencadenando distintas acusaciones: "Esta todo el curso dividido... se pelean siempre por la graduación” (Maxi, estudiante de 5to año. RNo 73, observación en clase de 5to año. 10/09/18); “iUn desastre! ni se sabe dónde lo vamos a hacer" (Valeria, estudiante de 5to año. RNo 83, entrevista. 05/11/18); “¡Siempre lo mismo! ¡Dan doscientas vueltas!” (RNo 87, observación. 26/11/18). Hasta que, finalmente, un grupo de estudiantes convocó a una asamblea, aunque sin la participación de las y los docentes ( $\mathrm{RN}$ o 87, observación en 5to año. 26/11/18).

Más allá de las acusaciones cruzadas habituales, la asamblea se efectuaba en un contexto especial. Desde hacía semanas, las clases de integración se habían destinado a intentar encausar las características que tendría el festejo, que siempre eran objeto de controversias. Como venía sucediendo en los últimos años, según me relataron en las entrevistas las y los graduados, en torno a la graduación se articulaban expectativas acerca "el vestido largo", “el traje” y el "lujo", arraigadas en la mayoría de los y las estudiantes: "la graduación era algo como para tomar más como de fieeesta... jasí! [Gesticula con los brazos abiertos] ¡Festejar alocadamente! [...] con todo el lujo” (Antonia, egresada. RNº 71, entrevista, 03/09/2018). 
Estas expectativas eran combatidas por las y los docentes, que buscaban, una y otra vez, "plantear críticamente el tema de la graduación. La noche de la graduación [enfatiza]... Como la noche de la princesa. Del príncipe (...) una herramienta del mercado" (Julieta, docente. RNo 93, entrevista. 01/03/2019). Lo que es más, en esta oportunidad se añadía el hecho de que habiendo obtenido la oficialización de la escuela, las y los docentes pretendían realizar un gran festejo en el mismo barrio, "que sean a la vez la entrega de libretas y la graduación, el festejo por la oficialización, todo junto. Invitando a mucha gente, los estudiantes, docentes anteriores, vecinos, otros partidos políticos" (Andrea, docente. $\mathrm{RN}$ o 80. 08/10/18).

A contramarcha de la intención de los/as docentes, sin embargo, los/as estudiantes habían decidido asumir la organización del festejo por su cuenta. Oponerse a la voz de los docentes no había sido sencillo, y las idas y vueltas a las que referían en sus quejas daba cuenta de las ambivalencias que había tenido el proceso de organizarse solos. Para sostener su posición debieron, forzosamente, componer asambleas, organizarse para recaudar el dinero necesario y arribar a acuerdos por sobre los conflictos, todo por cuenta propia. Durante las últimas semanas del año observé el trajín en el que se implicó una gran mayoría del curso, hasta que lograron concretar la graduación con las características que ellos deseaban: en un salón de alquiler, todos/as ataviados elegantemente, y con la cuantiosa presencia de invitados/as (RNo 97, festejo de graduación 15/12/18).

En una entrevista realizada pocas semanas antes del festejo, Valeria, estudiante de 5to, tomó como ejemplo este desenlace para ilustrar las diferencias que hallaba entre esta escuela y otras que había conocido. En su mirada, "haciéndolo solos", habían puesto en ejercicio el "compañerismo" que habían aprendido:

Vanesa: Acá aprendes otras cosas que en las escuelas... valores... que por ahí en otras escuelas no te enseñan. Que acá sí.

Marilín: ¿Como cuál? ¿Qué valor, por ejemplo?

Vanesa: Y, por ejemplo, insisten con el compañerismo. Que en sí, es verdad. Porque si no hay compañerismo no podés seguir. Y se dio con la graduación, que al final la terminamos haciéndolo solos, y termino muchísimo mejor que haciéndolo con los profes.

(Vanesa, 27 años, estudiante de 5to año. RNo 85, entrevista. 12/11/18).

Desde mi perspectiva, los sucesos relatados permiten ver cómo las prácticas asociadas a la gestión social van siendo apropiadas por las y los estudiantes, pero en articulación con sentidos y valoraciones propias, que pueden no coincidir o incluso oponerse a lo esperado por las y los docentes. En ese aprendizaje despliegan resignificaciones que implican una dimensión creativa, participando activamente de la producción de las prácticas y sentidos que se despliegan en la escuela.

\section{La gestíon social y el involucamiento político de las y los jovenes}

Como fue desarrollado en los apartados anteriores, el involucramiento en las actividades vinculadas a la gestión social de la escuela se va dando, en la práctica, como una construcción heterogénea, 
en la que las y los estudiantes se implican de modo dispar. A la vez, estas prácticas forman parte de un proceso de aprendizaje que se despliega en el transcurrir de la experiencia, al tiempo que las y los estudiantes van comprendiendo e "internalizando", como mencionaba Ariel, la propuesta escolar. En ese camino, algunos/as estudiantes se verán “transformados/as” y, como señala Carolina, se irán “involucrando en todo".

En el curso de ese aprendizaje, con todas sus tensiones y contradicciones, las y los estudiantes se van implicando en un hacer colectivo, en el cual la escuela misma adquiere existencia. Como destacaba Andrea (docente. RNo 68, entrevista. 27/08/18), "lo de gestión social muchos lo comprenden en cuanto que ven que el salón no se arma si ellos no están armados... que no se desarma si no lo hacen ellos". Es decir, sin la necesaria participación de las y los estudiantes, la escuela no se sostiene.

Por otro lado, es en ese mismo proceso, que se abren las posibilidades para la emergencia de lo político, y para el involucramiento de los estudiantes con el proyecto colectivo. Así, habitar la escuela de gestión social supone negociar, forzosamente, los compromisos prácticos con ese entorno (Massey 2005). Se trata de compromisos que se tejen en el mismo hacer, y cuyo significados, heterogéneos, se negocian y definen en el día a día (Fernández Álvarez 2015). Como vimos, esta participación nunca será total, y mientras algunas actividades son muy convocantes, como la organización de la graduación, otras recaen en un número relativamente pequeño de estudiantes: “[Siempre] éramos cinco o seis los que nos quedábamos al final para ordenar” (Antonia, 24 años, egresada. RNo 91, entrevista. 29/08/2019).

En la perspectiva de algunos/as de los egresados, lo que es más, habitar la escuela los inscribe en un conjunto de relaciones afectivas, con otros sujetos y con el lugar: "Acá no solo son tus profesores, sino que se tornan a ser tus compañeros, o entre comillas, como tus amigos" (Ariel, 21 años, egresado. RNo 45, entrevista, 10/12/18); "Yo de la escuela estaba enamorada... jenamorada de una institución! [Gesticula demostrando sorpresa, o incredulidad]. Por eso era como todo tan lindo. Es como que el amor te va moviendo" (Carolina, 20 años. RNo 94, entrevista. 16/08/19). Relaciones que, en sus palabras, los/as hace "sentirse parte": "Te hacen sentir parte... ¡de todo! de su vida, de la clase" (Antonia, 21 años, estudiante egresada. RNo 26, entrevista. 03/09/2018).

El "cuidado de la escuela”, como describía Analía (egresada. RNo 96, entrevista. 29/08/2019), "te retribuye, te hace bien" porque permite "sentir el espacio como propio". "Acá es como que vos ya conocés, es tu casa”, enfatiza más adelante en su relato. Al momento de la entrevista, la joven había terminado la escuela hacía ya cuatro años, pero continuaba yendo por las mañanas para colaborar en el jardín de infantes. Según sus palabras, esta relación con el lugar ya está "naturalizada": "Nosotras venimos acá, y es como... bueno: entramos acá, entramos allá, abrimos, nos tomamos mates, ponemos la pava, cebamos mate. Y eso no lo hacés en cualquier lado, no lo hacés en otra escuela. Es como otra casa”.

Actualmente, en el jardín, Analía se encarga de la coordinación de un grupo que se conformó con las madres de los niños y niñas, con el objetivo de que "las madres también sientan este espacio que es para sus hijos, de ellas también". "Para mí siempre el sentido fue integrar a las madres, que se sientan el espacio propio, para que se apropien de la institución, de la escuela. Lo mismo que pasa en la nocturna", describe. En su relato, las tareas de cuidado de la escuela cobran centralidad como parte de esta apropiación: 
Acá, viste, está lleno de tablones, lleno de cosas a la noche, pero después de eso, los cierran, los ponen allá, pasan el trapo de piso, dejan todo impecable, así, para que el jardín venga a la mañana. Y son todos los pibes y pibas que vienen a la noche, después de que terminan, guardan todo... la cocina, el patio... itodos los espacios son de todos! (Analía, egresada. RNo 96, entrevista. 29/08/2019).

En sentido contrario, es importante señalar que, si se tiene en cuenta el número global de estudiantes que han transitado por la escuela, quienes mantienen algún vínculo, ya sea asistiendo regularmente para colaborar, llevando a sus hijos/as al jardín de infantes, o al menos presentándose esporádicamente de visita, aquellos/as que conservan una participación activa y sostenida luego de egresar son efectivamente pocos/as.

Como sostiene Nespor (1994 apud Rockwell 2018:245-246), la escuela constituye un anudamiento temporal de relaciones entre sujetos, hasta cierto punto azaroso, que hace de la escuela un lugar de tránsito, "flojamente acoplado". Un anudamiento que, para una importante mayoría, se interrumpe cuando llega el momento del egreso. Sin embargo, es necesario dejar apuntado, en contra de otras interpretaciones, que la interrupción de la participación en la escuela no debe impedir considerar otros modos que puede asumir el involucramiento político, menos evidentes, o menos "conscientemente radicales", en términos de Gledhill (2000), pero no por ello menos significativos desde las experiencias de las y los sujetos.

En tal sentido, resulta sugerente recuperar el relato de Carolina, joven egresada. Reflexionando sobre su propio recorrido al finalizar la escuela, y algo desilusionada, detalla las dificultades y contratiempos que fue encontrando ante sus repetidos intentos de integrarse a otras actividades promovidas por la organización, y apuntala: "sentía que ya no era mi lugar. Y no por el hecho de que haya otros alumnos. Si no por el hecho de que... si yo ya terminé acá, iqué voy a venir a sentarme! Como que ya no me sentía parte de nada”.

Sin embargo, continuando con su narración, esgrime: "Ahora que no estoy militando, la escuela siempre la milito yo, desde mi parte”. Al repreguntar en la entrevista, y retomando su expresión, qué sería en su perspectiva "militar la escuela", define: "Soy mucho más de la palabra, y del contar, del convidar, de llevar gente a los lugares, que más gente conozca, que se hable más. Hacer conocidas las cosas”. Finalmente, mientras muestra en su brazo un tatuaje del logo de la escuela, remata: Yo la escuela la llevo toda entera a dónde voy. Es como... ¡es mi vida! no es parte de mi vida, es mi vida [enfatiza] iy la llevo a dónde sea!" (Carolina, egresada. RNo 94, entrevista. 16/08/2019).

A partir de esos pasajes es posible dejar abiertos interrogantes acerca de las huellas de las experiencias escolares que, incorporadas ya a la vida de estos/as jóvenes y abiertas a otras posibles reformulaciones, trasvasan el tiempo y espacio de la escuela. 


\section{Reflexiones finales}

En este trabajo exploré los procesos de construcción de una escuela para jóvenes y adultos/as, impulsada por una organización política en un contexto de desigualdad y pobreza urbana. Me propuse hacerlo desde la perspectiva de las y los jóvenes estudiantes, cuyas experiencias en la trama de estas particulares propuestas educativas han sido relativamente poco estudiadas.

Como quise demostrar, la escuela adquiere existencia cotidiana por medio y a través del despliegue de un hacer colectivo, que en ese universo social recibe un nombre preciso: gestión social. Aunque el involucramiento de las y los estudiantes con ese hacer nunca es total, y varía según las actividades de las que se trate, constituye una condición necesaria para el sostenimiento de la escuela.

En tanto categoría socialmente construida, la gestión social remite a un conjunto diverso de prácticas, que las y los estudiantes deben desentrañar en el día a día de la escuela. De este modo, al desplegar parte de la variedad de prácticas y sentidos que dicha categoría social designa, apunté a señalar, en primer lugar, las contradicciones que forman parte del aprendizaje de ese hacer y que dan cuenta, a la par, del rol activo que asumen las y los estudiantes en el proceso de aprender. Así, en la tarea de encontrar sentido a su experiencia cotidiana, y ante una propuesta escolar que perciben diferente y que no acaban de comprender, las y los estudiantes expresan distintas tensiones, y pueden resistirse de maneras más o menos explícitas.

En segundo lugar, describí los sentidos construidos por las y los estudiantes en torno al aprendizaje de la gestión social en el proceso escolar. Como parte de una experiencia formativa, estas prácticas van siendo apropiadas por las y los estudiantes, en articulación con sentidos y valoraciones propias. La descripción de las tensiones y conflictos en torno a la organización del festejo de graduación apuntó al modo en que estos aprendizajes son puestos en práctica, aún a contramarcha de la intención de las y los docentes.

Por último, de acuerdo a las vivencias relatadas por jóvenes graduados/as, las experiencias en torno a la gestión social se muestran como una vía para explorar los modos en que puede construirse el involucramiento con el proyecto colectivo. Haciendo, parte de las y los sujetos entablan relaciones con el espacio y entre sí, realizan inversiones afectivas que anudan trayectorias y las fijan, ocupando posiciones desde las cuales "sentirse parte" de la escuela. Sin embargo, y como contracara a la elocuencia con la que estos sentidos se expresan en ciertos casos, la gran mayoría de las y los egresados se desvinculan de la escuela al finalizar sus estudios.

Desde mi perspectiva, finalmente, el análisis desarrollado ofrece una vía de entrada para considerar la relación entre escuela y política desde la experiencia de las y los jóvenes estudiantes. A contramarcha de otras interpretaciones, en las que el sentido político de la propuesta educativa se comprende como derivación de la articulación entre las escuelas y las organizaciones que las gestan, o bien por el carácter de la relación que dichas organizaciones mantienen con respecto al Estado, propongo enfocar lo político como dimensión de la vida cotidiana, imbricada en la experiencia escolar, y como una construcción en la que los/as propios/as estudiantes tienen un lugar central. 
Marilin López Fittipaldi es Doctoranda en Antropologia, Facultad de Filosofía y Letras, Universidad de Buenos Aires (FFyL, UBA). Es Docente auxiliar de lera categoría en la Escuela de Antropologia (FHyA, UNR) y miembro del Programa de Antropología y Educación del Centro de Estudios Antropológicos en Contextos Urbanos (CEACU, FHyA, UNR). Es Becaria Doctoral del Consejo Nacional de Investigaciones Cientificas y Técnicas (CONICET).

\section{REFERENCIAS BIBLIOGRÁFICAS}

Achilli, Elena. 2005. Investigar en Antropología Social. Los desafios de transmitir un oficio. Rosario: Laborde.

Achilli, Elena. 2018. "Antropología y educación. Perspectivas, problemáticas y desafíos". In: Antropología Social. Perspectivas y problemáticas. v. 2. Santa Fe: Laborde.

Ampudia, Marina. 2013. "Educación y Autogestión. Estética Política y Autogestión. Estética política de los Bachilleratos Populares". In: Movimientos sociales, educación popular y trabajo autogestionado en el cono sur, ed. R. Elisalde, R. Falero, N. Dal Ri \& M. Ampudia. Buenos Aires: Buenos Libros.

Burgos, Aleandro, Nora Gluz e Mariel Karolinski. 2008. "Las experiencias educativas de los movimientos sociales: Reflexiones en torno a la construcción de autonomía”. Pp 171-95 in V Jornadas de Sociología de la UNLP. Recuperado de: https://www.aacademica.org/000-096/303.pdf

Caisso, Lucía. 2014. Una escuela como ésta. Experiencias educativas en un movimiento social de la ciudad de Córdoba (Argentina). Tesis Doctoral. Universidad Nacional de Córdoba.

Caisso, Lucía. 2017. "Educación popular, educación tradicional: análisis etnográfico de un conflicto en un bachillerato popular". Etnografica 21 (2): 341-364. doi: https://doi.org/10.4000/etnografica. 4933

Cañedo Rodriguez, Montserrat. 2011. Antropología politica: temas contemporáneos. Barcelona: Bellaterra.

Cerletti, Laura e Laura Santillán. 2015. “Lo 'histórico’ en la investigación etnográfica: las presencias de múltiples temporalidades, dificultades y desafíos”. Boletín de Antropología y Educación 9(9):115120. Recuperado de: http://antropologia.institutos.filo.uba.ar/sites/antropologia.institutos.filo.uba. ar/files/bae_n09_e02a03.pdf

Consejo Federal De Educación. 2007. Resolución No33/07y Anexo 01 Escuelas de Gestión Social. Recuperado de: http://www.me.gov.ar/consejo/resoluciones/res07/33-07-anexo01.pdf 
Elisalde, Roberto. 2008. “Movimientos sociales y educación: Bachilleratos Populares en empresas recuperadas y organizaciones sociales. Experiencias pedagógicas en el campo de la educación de jóvenes y adultos". In: Movimientos sociales y educación. Teoría e historia de la Educación Popular en Argentina y América Latina, ed.R. Elisalde y M. Ampudia. Buenos Aires: Buenos Libros.

Fernández Álvarez, María Inés. 2015. “Contribuciones antropológicas al estudio de las cooperativas de trabajo en la Argentina reciente". Revista del Centro de Estudios de Sociología del Trabajo (CESOT) 7:37-64. Recuperado de: https://ojs.econ.uba.ar/index.php/CESOT/article/view/1438

García, Javier. 2011. Aprendiendo a hacer escuelas. Las complejas y dinámicas relaciones entre 'Bachilleratos Populares'y Estado. Tesis de Maestría. Universidad de Buenos Aires. Recuperado de: http:// repositorio.filo.uba.ar/bitstream/handle/filodigital/1741/uba_ffyl_t_2011_se_garcia.pdf?sequence $=3 \&$ is Allowed $=y$

García, Javier. 2018. La producción cultural del sujeto critico : construcciones de conocimientos en "Bachilleratos Populares". Tesis Doctoral.Universidad de Buenos Aires. Recuperado de: http://repositorio.filo.uba.ar/bitstream/handle/filodigital/9989/uba_ffyl_t_2018_se_garciaja.pdf ?sequence=2\&isAllowed $=y$

Giarracca, Norma; Miguel Teubal. 2007. “La rebelión de 2001: protestas, rupturas y recomposiciones". In: Tiempos de rebelión: "que se vayan todos". Calles y plazas en la Argentina 2001-2002, N. Giarracca, D. Mariotti \& M. Comelli. Buenos Aires: Antropofagia.

Gledhill, John. 2000. El poder y sus disfraces. Perspectivas antropológicas de la política. Barcelona: Bellaterra.

Gluz, Nora. 2013. "Alternativas escolares en movimientos sociales y procesos de subjetivación política”. X Jornadas de Sociología. Recuperado de: https://cdsa.aacademica.org/000-038/606.pdf

Grupo De Estudios Sobre Movimientos Sociales Y Educación Popular (GEMSEP). 2016. Informe relevamiento Nacional de Bachilleratos Populares de Jóvenes y Adultos. Recuperado de: https://drive. google.com/file/d/0B9WAEryqfqZ5MUd4OHRQM2NPMFk/view

Ingold, Tim. 1993. “The temporality of the landscape”. World Archaeology 25(2):152-74. doi: https://doi.org/10.1080/00438243.1993.9980235

Kriger, Miriam e Shirly Said. 2015. “Subjetivación política juvenil en Bachilleratos Populares: representaciones de estudiantes jóvenes sobre la política y el Estado”. Pp1-19 in XI Jornadas de Sociología. Recuperado de: https://www.aacademica.org/000-061/885

Kriger, Miriam e Shirly Said. 2017. "Hacer política desde la escuela: narrativas biográficas de jóvenes en Argentina”. Revista latinoamericana de ciencias sociales, niñez y juventud 15(2):1085-96. doi: http://dx.doi.org/10.11600/1692715x.1521920122016 
Ley De Educación Nacional N² 26.206. 2006. Recuperado de: http://www.me.gov.ar/doc_pdf/ ley_de_educ_nac.pdf

López Fittipaldi, Marilín. 2015. Movimientos sociales y educación. Un análisis antropológico del proceso de construcción de un Bachillerato Popular en la ciudad de Rosario. Licenciatura. Universidad Nacional de Rosario.

Manzano, Virginia. 2016. “Tramas de bienestar, membresía y sujetos políticos: La Organización Tupac Amaru en el norte argentino" Revista Ensambles 3:54-71. Recuperado de: http://www.revistaensambles.com.ar/ojs-2.4.1/index.php/ensambles/article/view/74/49

Manzano, Virginia e Ana Ramos. 2015. "Procesos de movilización y de demandas colectivas: estudios y modos de abordar 'lo político' en la vida social”. Identidades 5(8):1-25.Recuperado de: https:// ri.conicet.gov.ar/handle/11336/54676

Massey, Doreen. 2005. For space. Londres: Sage Publications.

Núñez, Pedro e Lucía Litichever. 2016. "Ser joven en la escuela: temporalidades y sentidos de la experiencia escolar en la Argentina”. Psicoperspectivas. Individuo y Sociedad 15(2): 90-101.doi: http:// dx.doi.org/10.5027/psicoperspectivas-Vol15-Issue2-fulltext-854

Peirano, Mariza. 2002. "Rituais como estratégia analitica e abordagem etnográfica”. In:O Dito e o feito. Ensaios de antropologia dos rituais. Río de Janeiro: Relumé Dumará, Núcleo de Antropologia da Política/UFRJ.

Quirós, Julieta. 2014. “Etnografiar mundos vívidos. Desafíos de trabajo de campo, escritura y enseñanza en antropología”. Publicar XII(XVII):47-65. Recuperado de: http://ppct.caicyt.gov.ar/index.php/publicar/article/view/4914

Rockwell, Elsie. 2000. “Caminos y rumbos de la investigación etnográfica en América Latina”. Cuadernos De antropología Social (13):53-64. doi: https://doi.org/10.34096/cas.i13.4664

Rockwell, Elsie. 2012. "Movimientos sociales emergentes y nuevas maneras de educar". Educação e Sociedade 33(120):697-713. doi: https://doi.org/10.1590/S0101-73302012000300003

Rockwell, Elsie. 2018a. "Los niños en los intersticios de la cotidianeidad escolar: ¿̨resistencia, apropiación o subversión?" In: Vivir entre escuelas. Relatos y presencias. Buenos Aires: CLACSO. Recuperado de: http://biblioteca.clacso.edu.ar/clacso/se/20180223024326/Antologia_Elsie_Rockwell.pdf Rockwell, Elsie. 2018b. "Temporalidad y cotidianeidad en las culturas escolares". Cuadernos de Antropología Social 47:21-32. doi: https://doi.org/10.34096/cas.i47.4945 
Said, Shirly. 2018. "Young people in Popular High Schools : Between individuation and political subjectivation". Universitas. Revista de Ciencias Sociales y Humanas (28):141-57. doi: http://dx.doi. org/10.17163/uni.n28.2018.07

Santillán, Laura. 2015. "La demanda por educación en barrios populares del Gran Buenos Aires: acerca de la militancia política, el voluntariado social y la actuación colectiva de los pobladores (1985 y 2015)”. In: XI Reunión de Antropología del MERCOSUR. Montevideo, Uruguay.

Santillán, Laura. 2019. “'Nuestro norte son los niños’ Subjetividades políticas y colectivización del cuidado infantil en organizaciones sociales del Gran Buenos Aires”. Runa 9628:57-74. doi: https:// doi.org/10.34096/runa.v40i2.6282

Sirvent, María Teresa. 2005. "La educación de jóvenes y adultos frente al desafío de los movimientos sociales emergentes en Argentina". Revista Brasileira de Educação (28):37-49. doi: https://doi. org/10.1590/S1413-24782005000100004

Svampa, Maristella e Sebastián Pereyra. 2003. Entre la ruta y el barrio. La experiencia de las organizaciones piqueteras. Buenos Aires: Biblos.

Torres Carrillo, Alfonso. 2017. "Prácticas educativas en movimientos sociales de América Latina”. Folios 46:3-14. doi: https://doi.org/10.17227/01234870.46folios3.14

Weiss, Eduardo. 2015. "Más allá de la socialización y de la sociabilidad: Jóvenes y bachillerato en México”. Educação e Pesquisa 41(Special issue):1257-72. doi: https://doi.org/10.1590/S15179702201508144889 


\title{
“FAÇA ESCOLA, FAÇA COLETIVO”. MOVIMENTOS SOCIAIS, JUVENTUDE E EXPERIÊNCIAS EDUCACIONAIS DESDE UMA PERSPECTIVA ANTROPOLÓGICA (ROSÁRIO, ARGENTINA)
}

Resumo: Neste artigo, proponho analisar a produção coletiva de uma escola para jovens e adultos, promovida por uma organização política em um contexto de desigualdade e pobreza urbana, na cidade de Rosário, Argentina. Os dados empíricos nas quais a análise se baseia foram construídas a partir de um trabalho de campo etnográfico que venho realizando de 2011 até o presente. Destaco o caráter da construção inacabada e permanente que os projetos educacionais emergentes têm, ao mesmo tempo em que aponto a contribuição central que os jovens estudantes dão a esse processo. A análise busca documentar, por meio da exploração da categoria nativa "gestão social", a experiência dos alunos no fazer coletivo da escola. Ao contrário de outras interpretações, abordo o político não como uma dimensão externa, mas inserida no cotidiano da experiência escolar, uma construção na qual os próprios alunos ocupam um lugar central.

Palavras-chave: Movimentos sociais; Experiências educacionais; Juventude; Gestão social; Envolvimento político.

\section{“MAKE SCHOOL, MAKE COLLECTIVE”. SOCIAL MOVEMENTS, YOUTH AND EDUCATIONAL EXPERIENCES FROM AN ANTHROPOLOGICAL PERSPECTIVE (ROSARIO, ARGENTINA)}

\begin{abstract}
In this article, I propose to analyze the collective production of a school for youth and adults, promoted by a political organization in a context of inequality and urban poverty, in the city of Rosario, Argentina.The empirical data comes from an ethnographic field work that I have been carrying out from 2011 to the present. I highlight the character of unfinished and permanent construction that the emerging educational projects have, as well as I point out the key contribution that young students make in this type ofprocess. The analysis seeks to document, through the exploration of the native category "social management", the experience of students in the collective making of the school. Unlike other interpretations, I approach the political not as an external dimension, but embedded in the everyday life of the school experience, a construction in which students themselves have a central place.
\end{abstract}

Keywords: Social movements; Educational experiences; Youth; Social management; Political involvement.

RECEBIDO: $30 / 04 / 2020$

APROVADO: $08 / 01 / 2021$ 